\title{
Interpersonal therapy alone and supportive therapy plus antidepressant drugs were most effective for depression in HIV positive patients
}

\author{
Markowitz JC, Kocsis JH, Fishman B, et al. Treatment of depressive symptoms in human immunodeficiency virus-positive patients. \\ Arch Gen Psychiatry 1998 May;55:452-7.
}

\section{Question}

In HIV positive patients, is interpersonal psychotherapy or cognitive behavioural therapy effective in treating depressive symptoms compared with supportive psychotherapy with or without pharmacotherapy?

\section{Design \\ 16 week randomised controlled trial.}

\section{Setting}

A university centre in New York, New York,USA.

\section{Patients}

101 patients (mean age $37 \mathrm{y}, 85 \%$ men) who were HIV positive for $\geqslant 6$ months, had a score of $\geqslant 15$ on the 24 item Hamilton Depression Rating Scale (HAM-D), and were physically well enough to permit outpatient treatment. Exclusion criteria were substantial non-HIV related disease, schizophrenia, bipolar disorder, allergy to imipramine, substance abuse, cognitive impairment, inability to speak English, or concurrent psychiatric treatment for other reasons.

\section{Intervention}

Patients were allocated to cognitive behavioural therapy (CBT) $(n=27)$, interpersonal psychotherapy (IPT) $(n=24)$, supportive psychotherapy (SP) $(n=24)$, or supportive psychotherapy plus imipramine (SWI) $(n=26)$. CBT and IPT focused on specific themes whereas SP was less structured, giving psychoeducation about depression and HIV but no framework for therapy. In the SWI group imipramine was given at $50 \mathrm{mg} /$ day and increased to $300 \mathrm{mg}$ /day for 3 to 4 weeks if tolerated.

\section{Main outcome measures}

Mean scores on the 24 item HAM-D, Beck Depression Inventory (BDI), and 100 point Karnofsky scale; and CD4 cell count.

\begin{abstract}
Main results
Using intention to treat analysis, all groups showed improvement for HAM-D and BDI scores by 16 weeks. The HAM-D scores at 16 weeks for IPT and SWI were both superior to CBT. The mean scores were 10.6 for IPT, 11.8 for SWI, 15.5 for SP, and 17.1 for CBT $(p=0.05$ for IPT and SWI compared with CBT). The mean BDI scores for SWI, IPT, CBT, and SP were 11.7, 14.0, 19.8, and 20.3, respectively. SWI was more effective than CBT and SP $(\mathrm{p}=0.007)$ and IPT was more effective than SP $(p=0.007)$. The groups did not differ when adjustment was made for CD4 cell count and Karnofsky physical functioning score. Karnofsky scores increased from baseline in all 4 groups but, at study end, higher mean Karnofsky scores were seen in the IPT and SWI groups than in the CBT and SP groups (90.9 and $88.3 v 83.1$ and $82.5, \mathrm{p}=0.006)$. The $\mathrm{CD} 4$ cell count did not substantially change across groups.
\end{abstract}

\section{Conclusion}

Interpersonal psychotherapy was comparable to supportive psychotherapy plus pharmacotherapy, and was more effective than cognitive behavioural therapy or supportive therapy alone for treating depressive symptoms in HIV positive patients.

Sources of funding: National Institute of Mental Health and the New York Community Trust by DeWitt-Wallace.

For correspondence: $\operatorname{Dr} J$ C Markowitz, Cornell University Medical College, 445 East 68th Street, Suite 3N, New York, NY 10021, USA.Email jcmarko@mail.med.cornell.edu

\section{Commentary}

People with HIV infection are at increased risk of psychological disturbance, but there have been few investigations of the efficacy of psychological and other treatments in these patients. ${ }^{1}$ Markowitz et al have done an important study which should help the development of more evidence-based interventions.

It is important to stress that patients in all 4 groups improved significantly, with no differences in the proportion of patients in remission from depression, although some treatments, in particular IPT and SWI, had more rapid and stronger effects. Although it is important to discuss the relative performance of IPT and CBT, it would be wrong to ignore the evidence for the impressive efficacy of SWI, a treatment more easily accessible than IPT or CBT.
The relatively poor performance of CBT is somewhat puzzling but is consistent with other studies which have not shown it to be superior to other psychological interventions in this patient group. ${ }^{23}$ It is possible, as argued by the authors, that IPT is more appropriate for people facing life events and changes in their life roles. On the other hand, CBT may lead to the development of a slower and more persistent change in ways of thinking, with sustained longer term gains. The short period of evaluation (16 weeks) and the absence of a reasonable follow up prevent testing of this possibility.

In practice, the study shows that, in the short term, supportive psychotherapy plus antidepressants are at least as effective as more complex psychotherapeutic treatments for people with HIV and depressive symptoms. No evidence is available for long term efficacy or to decide what treatment is appropriate for whom. The impressive performance of IPT suggests that training and research using this treatment modality should become more widely available.

Jose Catalán, MSc, DPM, FRCPsych Imperial college School of Medicine

London, $U K$

1 Catalán J, Burgess A, Klimes I. Psychological Catalán J, Burgess A, Klimes I. Psychological medicine of HIV infecion

2 Auerbach J, Oleson T, Solomon G. A behavioural medicine intervention as an adjuvant treatment for HIV-related illness. Psychology and Health 1992;6:325-34.

3 Kelly JA, Murphy DA, Baht GR, et al. Outcome of cognitive-behavioral support group brief therapies for depressed, HIV-infected persons. Am J Psychiatry. 1993;150:1679-86. 